Korean J. Math. 20 (2012), No. 4, pp. 423-431

http://dx.doi.org/10.11568/kjm.2012.20.4.423

\title{
MONOIDS OVER WHICH ALL REGULAR RIGHT S-ACTS ARE WEAKLY INJECTIVE
}

\author{
Eunho L. MoON
}

\begin{abstract}
There have been some study characterizing monoids by homological classification using the properties around projectivity, injectivity, or regularity of acts. In particular Kilp and Knauer([4]) have analyzed monoids over which all acts with one of the properties around projectivity or injectivity are regular. However Kilp and Knauer left over problems of characterization of monoids over which all regular right $S$-acts are (weakly) flat, (weakly) injective or faithful. Among these open problems, Liu([3]) proved that all regular right $S$-acts are (weakly) flat if and only if $e s$ is a von Neumann regular element of $S$ for all $s \in S$ and $e^{2}=e \in T$, and that all regular right $S$-acts are faithful if and only if all right ideals $e S$, $e^{2}=e \in T$, are faithful. But it still remains an open question to characterize over which all regular right $S$-acts are weakly injective or injective. Hence the purpose of this study is to investigate the relations between regular right $S$-acts and weakly injective right $S$ acts, and then characterize the monoid over which all regular right $S$-acts are weakly injective.
\end{abstract}

Received September 10, 2012. Revised December 7, 2012. Accepted December 10,2012 .

2010 Mathematics Subject Classification: 20M10.

Key words and phrases: regular right $S$-act, weakly injective right $S$-act, von Neumann regular monoid.

(c) The Kangwon-Kyungki Mathematical Society, 2012.

This is an Open Access article distributed under the terms of the Creative commons Attribution Non-Conercial License (http://creativecommons.org/licenses/bync/3.0/) which permits unrestricted non-commercial use, distribution and reproduction in any medium, provided the original work is properly cited. 


\section{Introduction}

In module theory three different definitions of a regular module have been introduced. One of them is defined by Ware for which a regular module is projective such that all its cyclic submodules are direct summands $([6])$. A bigger class of regular modules is defined by Zelmanowitz([7]).

Extending the notion of von Neumann regularity of semigroup, Tran([5]) introduced the definition of a regular act over a momoid $S$ as follow: A right $\mathrm{S}$-act $A$ is called regular if for any element $a \in A$ there is an $S$ homomorphism $f: a S \rightarrow S$ such that $a f(a)=a$. This definition of a regular right $\mathrm{S}$-act is similar to the one of Zelmanowitz for modules and keeps regular acts close to projective acts and to von Neumann regular monoids.

We note that if $S$ is the von Neumann regular monoid, then for every $s \in S$ there is an element $s^{\prime} \in S$ such that $s s^{\prime} s=s$, hence the $S$-homomorphism $f: s S \rightarrow S$ defined by $f(s t)=s^{\prime} s t, t \in S$ satisfies $s f(s)=s s^{\prime} s=s$. Thus the von Neumann regular monoid $S$ is a regular right S-act. But a monoid $S$ which is a regular right $S$-act need not be von Neumann regular. For example, the semigroup $N$ of all natural numbers is not von Neumann regular but a regular $N$-act.

Throughout this paper, $S$ will stand for a monoid and every act will denote a right $\mathrm{S}$-act over a monoid $S$. A right $S$-act is a set $A$ on which $S$ acts unitarily from the right in the usual way, that is to say

$$
a(s t)=(a s) t, \quad a 1=a, \quad a \in A, \quad s, t \in S
$$

where 1 denotes the identity of $S$.

We shall give useful characterizations of a regular act as follow:

Theorem 1. ([5]) For a right $S$-act $A$, the following conditions are equivalent:

(i) $A$ is regular.

(ii) for every $a \in S$ there is an idempotent $\lambda_{a} \in S$ such that $a S$ is isomorphic to $\lambda_{a} S$.

(iii) for every element $a \in A$ aS is a projective $S$-act. 
Note: From theorem 1 every $S$-act is regular if and only if $S=\{1\}$ or $S=\{0,1\}$.([5])

In general regularity of an act does not imply projectivity and also projectivity does not imply regularity, hence Tran([5]) has investigated monoids over which the class of regular acts coincides with the class of projective acts as follows:

Lemma 1. A monoid $S$ is a regular right $S$-act if and only if every projective right $S$-act is regular.

ThEOREM 2. For a monoid $S$ the following conditions are equivalent:

(i) $S$ is a group.

(ii) an $S$-act $A$ is regular if and only if $A$ is projective.

Moreover there has been done some study characterizing monoids by homological classification using the properties around projectivity, injectivity, or regularity of acts. In particular Kilp and Knauer([4]) have analyzed monoids over which all acts with one of the properties around projectivity or injectivity are regular as follows:

THEOREM 3. The following conditions on a monoid $S$ are equivalent:

(i) all principally weakly flat $S$-acts are regular.

(ii) all torsion free $S$-acts are regular.

(iii) all cofree $S$-acts are regular.

(iv) all injective cogenerators in $S$-act are regular.

(v) all injective $S$-acts are regular.

(vi) all weakly injective $S$-acts are regular.

(vii) all principally weakly injective $S$-acts are regular.

(viii) all divisible $S$-acts are regular.

(ix) all faithful $S$-acts are regular.

(x) all $S$-acts are regular.

(xi) $S=\{1\}$ or $S=\{0,1\}$.

Conversely Kilp and Knauer([4]) also have characterized monoids over which all regular acts have one of these properties. For example, it is proved that all regular right $S$-acts are projective $\Leftrightarrow$ all regular right $S$ acts are strongly flat $\Leftrightarrow$ all regular right $S$-acts are completely reducible $\Leftrightarrow$ every idempotent of $T$ generates a minimal right ideal where $T$ is the largest regular right ideal of $S$. Moreover, it is also proved that all 
regular right $S$-acts are free $\Leftrightarrow$ all regular right $S$-acts are projective generators in $S$-act $\Leftrightarrow \mathrm{S}$ is a group.

However Kilp and Knauer remained the problems of characterization of monoids over which all regular right $S$-acts are (weakly) flat([2]), (weakly) injective or faithful. Among these open problems, Liu([3]) proved that all regular right $S$-acts are (weakly) flat if and only if es is a von Neumann regular element of $S$ for all $s \in S$ and $e^{2}=e \in T$, and that all regular right $S$-acts are faithful if and only if all right ideals $e S, e^{2}=e \in T$, are faithful. But it still remains an open question to characterize over which all regular right $S$-acts are weakly injective or injective. Hence the purpose of this study is to investigate the relations between regular right $S$-acts and weakly injective right $S$-acts, and then characterize the monoid over which all regular right $S$-acts are weakly injective.

\section{Monoids over which all regular right S-acts are weakly injective}

A right $S$-act $A$ is called injective if given a $S$-monomorphism $i: M \rightarrow$ $N$ and any $S$-homomorphism $f: M \rightarrow A$, where $M, N$ are right $S$-acts, there exists a $S$-homomorphism $g: N \rightarrow A$ such that $f=g i$. A $S$-act $A$ is called (principally) weakly injective if for any inclusion $i: I \rightarrow S$ where $I$ is a (principal) right ideal of $S$ and for any $S$-homomorphism $f: I \rightarrow A$ there exists a $S$-homomorphism $g: S \rightarrow A$ such that $f=g i$. A $S$-act $A$ is called divisible if $A s=A$ for every left cancellable element $s$ of $S$.

REMARK. For any (right) $S$-act $A$, we have the following implications: $A$ is free $\Rightarrow A$ is a projective generator $\Rightarrow A$ is projective $\Rightarrow A$ is strongly flat $\Rightarrow A$ is flat $\Rightarrow A$ is weakly flat $\Rightarrow A$ is principally weakly flat $\Rightarrow A$ is torsion free.

$A$ is cofree or injective cogenerator $\Rightarrow A$ is injective $\Rightarrow A$ is weakly injective $\Rightarrow A$ is principally weakly injective $\Rightarrow A$ is divisible.

From above implications of (right) $S$-acts, we note that every (principally) weakly injective right $S$-act is divisible, but the divisible right 
$S$-act is not necessarily (principally) weakly injective. Hence we investigate the condition so that the class of (principally) weakly injective right $S$-acts coincides with the class of divisible right $S$-acts. For this, we shall characterize the left cancellative monoid by these properties of acts.

ThEOREm 4. Let $S$ be a left cancellative monoid. Then a right $S$-act $A$ is weakly injective if and only if $A$ is divisible.

Proof. It suffices to prove a divisible right $S$-act is principally weakly injective. Assume that $S$ is a left cancellative monoid and let $A$ be a right $S$-act and $I$ be a right ideal of a monoid $S$ and for any $a \in S$ and $x \in I$, let $f: I \rightarrow A$ be an $S$-homomorphism given by $f(x)=a$. If $A$ is divisible, then there is an $b \in A$ such that $a=b x$. Thus we define the map $g: S \rightarrow A$ by $g(1)=b$, then $g$ is clearly a well defined $S$-homomorphism with $g(x)=g(1) x=b x=a=f(x)$. Since $g$ is the extension of $f$ to $S, A$ is weakly injective.

Kilp and Knauer([4]) proved that all regular right $S$-acts are divisible if and only if all right ideals $e S, e^{2}=e \in T$ are divisible. Thus we first investigate necessary and sufficient condition so that all regular right acts over a left cancellative monoid $S$ are weakly injective.

TheOREm 5. Let $S$ be a left cancellative monoid. Then all regular right $S$-acts are weakly injective if and only if $S$ is a group.

Proof. $(\Rightarrow)$ : Assume that a monoid $S$ is left cancellative and all regular right $S$-acts are weakly injective and let $A$ be a regular right $S$-act. Then $A$ is weakly injective by assumption and so it is clearly divisible. Hence all regular $S$-acts are divisible and then $e S=S$ is divisible. Thus for any $x \in S$ there is $y \in S$ such that $1=y x$ and then it implies $y=1 y=y x y$ so that $x y=1$. Since for every $x \in S$ there is some $y \in S$ such that $1=x y=y x, S$ is a group.

$(\Leftarrow)$ : Assume that $S$ is a group and let $A$ be a right $S$-act. Then $A$ is the direct sum of cyclic subacts. In particular if $A$ is regular, then $A$ is projective. Moreover since $S$ is left cancellative, it has only one idempotent so that $A$ is free, that is, $A$ is isomorphic to the direct sum of copies of $S$. Moreover since $S$ has no proper right ideals, it is clear that any $S$-homomorphism $f: S \rightarrow A$ can be extended to $g: \oplus S \rightarrow A$ 
so that $A$ is weakly injective. Thus all regular right $S$-acts over a group $S$ are weakly injective.

Theorem 6. $S$ is a von Neumann regular monoid if and only if all right $S$-acts are principally weakly injective.

Proof. Let $A$ be an $S$-act and for any $s$ in $S$, let $f: s S \rightarrow A$ be an $S$-homomorphism. If $S$ is von Neumann regular, then there is an element $s^{\prime}$ in $S$ such that $s=s s^{\prime} s$. Set $f\left(s s^{\prime}\right)=a$ and define a mapping $g: S \rightarrow A$ by $g(1)=a$. Then $g$ is a well defined $S$-homomorphism and $g(s)=g(1) s=a s=f\left(s s^{\prime}\right) s=f(s)$. Since $g$ is the extension of $f$ to $S, A$ is principally weakly injective. Conversely assume that all right $S$-acts are principally weakly injective. Then for every $a$ in $S$, the principal right ideal $a S$ of $S$ is a principally weakly injective right $S$ act. Hence the identity map $i d$. of $a S$ into $a S$ can be extended to the $S$-homomorphism $g$ of $S$ onto $a S$. Set $g(1)=a a^{\prime}$ for some $a^{\prime}$ in $S$. Then $a=i d .(a)=g(a)=g(1) a=a a^{\prime} a$ so that $a$ is von Neumann regular.

A right $S$-act $A$ is called flat if the functor $A \otimes-$ preserves all monomorphisms. $A$ is called (principally) weakly flat if $A \otimes-$ preserves embeddings of (principal) left ideals of $S$ into $S$. $A$ is said to satisfy condition $(E)$ if whenever as $=a t$ for $a \in A, s, t \in S$ then there is $b \in A$ and $u \in S$ such that $u s=u t$ and $a=b u$.

Suppose that $I$ is a proper right ideal of $S$. If $x, y$ and $z$ are symbols not representing elements of $S$, define

$$
A(I)=(S-I,\{x, y\}) \cup(I,\{z\}),
$$

and define a right $S$-act on $A(I)$ by $(s, u) t=(s t, u)$ if $s t \notin I$ and $(s, u) t=(s t, z)$ if $s t \in I$ where $u \in\{x, y\}$ and $(s, z) t=(s t, z)$. Then $A(I)$ is a right $S$-act, and $A(I)$ satisfies condition $(E)$ for any proper right ideal $I$ of $S$.

Lemma 2. ([1]) Let I be a proper right ideal of $S$. Then the followings are equivalent:

(i) $A(I)$ is flat.

(ii) $A(I)$ is weakly flat.

(iii) $A(I)$ is principally weakly flat.

(iv) for every $a \in I, a \in I a$. 
We have that flatness implies weak flatness and weak flatness implies principal weak flatness. But $\operatorname{Liu}([1])$ showed that condition $(E)$ does not imply flatness, weak flatness or principal weak flatness. Also Liu characterized those monoids over which this implication does hold.

Theorem 7. ([1]) The followings are equivalent on a monoid $S$;

(i) $S$ is von Neumann regular.

(ii) all right $S$-acts satisfying condition $(E)$ are flat.

(iii) all right $S$-acts satisfying condition $(E)$ are weakly flat.

(iv) all right $S$-acts satisfying condition $(E)$ are principally weakly flat.

(v) all right $S$-acts are principally weakly flat.

TheOREM 8. The following statements are equivalent:

(i) $S$ is a von Neumann regular monoid.

(ii) every right ideal of a monoid $S$ is generated by an idempotent.

(iii) every right $S$-act is weakly injective.

Proof. (ii) $\Leftrightarrow$ (iii): Assume that every right ideal of a monoid $S$ is generated by an idempotent and let $A$ be a right $S$-act and $I$ be a right ideal of $S$. Then there is an idempotent $e$ of $S$ such that $I=e S$. If $f: I \rightarrow A$ is an $S$-homomorphism, then for every $s \in I, f(s)=f(e s)=$ $f(e) s$. We set $f(e)=a$ and define the map $g: S \rightarrow A$ by $g(1)=a$. Then $g$ is a well defined $S$-homomorphism and $g(s)=g(1) s=a s=f(e) s=$ $f(e s)=f(s)$. Hence $A$ is weakly injective. Conversely assume that all right $S$-acts are weakly injective, and let $I$ be a right ideal of $S$. Since $I$ is a right $S$-act, it is weakly injective by assumption. Hence the identity map $i d .: I \rightarrow I$ can be extended to a map $g: S \rightarrow I$. We set $g(1)=a$, then for every $s \in I, s=i d .(s)=g(s)=g(1) s=a s$. Thus $a=a^{2}$ and $I=a I \subset a S \subset I$ so that $I=a S$

$(i) \Leftrightarrow($ iii $)$ : Let $I$ be a right ideal of $S$ and consider the right $S$-act $A(I)$ satisfying condition $(E)$. If $S$ is von Neumann regular, then $A(I)$ is flat and hence for every $a \in I, a \in I a$, that is, there is some $b \in I$ such that $a=b a$. For any right $S$-act $M$ and an $S$-homomorphism $f: I \rightarrow M$, define a map $g: S \rightarrow M$ by $g(1)=f(b)$. Clearly $g$ is a well defined $S$-homomorphism with $g(a)=g(1) a=f(b) a=f(b a)=f(a)$. Hence $g$ is the extension of $f$ so that $M$ is weakly injective. Conversely if every right $S$-act is weakly injective, then every right ideal of $S$ is generated by an idempotent, hence for every $s \in S$, the principal right ideal $s S$ is generated by an idempotent, that is, there is an idempotent $e$ of $S$ such 
that $s S=e S$. If $e s=s$ and $e=s s^{\prime}$ for some $s^{\prime} \in S$, then $s=e s=s s^{\prime} s$ so that $s$ is von Neumann regular.

Next theorem gives a characterization of monoids over which all regular right $S$-acts are principally weakly injective.

THEOREM 9. ([4]) All regular right $S$-acts are principally weakly injective if and only if the largest regular right ideal $T \subset S$ is von Neumann regular and whenever $p \in S \neq T$ is e-cancellable for $e^{2}=e \in T$, then $e \in p S$.

We characterize the monoid over which all regular right $S$-acts are weakly injective.

Theorem 10. A monoid $S$ is a regular right $S$-act and all regular right $S$-acts are weakly injective if and only if $S$ is a von Neumann regular monoid.

Proof. Assume that $S$ is a regular right $S$-act and all regular right $S$-acts are weakly injective. Let $T$ be the union of all regular right ideals of $S$. If all regular right $S$-acts are weakly injective, then they are principally weakly injective so that $T \subset S$ is von Neumann regular by theorem 9 . Let $p \in S-T$. If $S$ is a regular $S$-act, then there is an idempotent $e \in S$ such that $h: p S \rightarrow e S$ is an isomorphism. Since $e S$ is regular, it is weakly injective and then there is an $S$-homomorphism $g: S \rightarrow e S$ such that $g$ is an extension of $h$. Hence $e=h(p)=g(p)=$ $g(1) p$ and then $p=p e=p g(1) p$ so that $p$ is also von Neumann regular. Thus $S$ is a von Neumann regular monoid.

For the converse, consider the right $S$-act $A(I)$ satisfying condition $(E)$. If $S$ is a von Neumann regular monoid, then $A(I)$ is flat and then for every $a \in I, a \in I a$ by lemma 2 . Hence for every $a \in I$, there is some $b \in I$ such that $a=b a$. For any right $S$-act $M$ and an $S$-homomorphism $f: I \rightarrow M$, we define the map $g: S \rightarrow M$ by $g(1)=f(b)$. Then for every $a \in I, g(a)=g(1) a=f(b) a=f(b a)=f(a)$ so that $M$ is weakly injective. Also a von Neumann regular monoid $S$ is clearly a regular right $S$-act.

\section{References}

[1] Liu Zhongkui, A characterization of regular monoids by flatness of left acts, Semigroup Forum 46 (1993), 85-89. 
[2] Liu Zhongkui, Monoids over which all regular left acts are flat, Semigroup Forum 50 (1995), 135-139.

[3] Liu Zhongkui, Monoids over which all flat left acts are regular, J. Pure Appl. Algebra 111 (1996), 199-203.

[4] Mati Kilp and Ulrich Knauer, Characterization of monoids by properties of regular acts, J. Pure Appl. Algebra 46 (1987), 217-231. J. of Pure and Applied Algebra 46 (1987), 217-231.

[5] Tran Lam Hach, Characterizations of monoids by regular acts, Period. Math. Hungar. 16 (4) (1985), 273-279.

[6] R. Ware, Endomorphism rings of projective modules, Trans. Amer. Math. Soc. 155 (1971), 233-256.

[7] J. Zelmanowitz, Regular modules, Trans. Amer. Math. Soc. 163 (1972), 341-355.

BangMok College of Basic Studies

Myongji University

Kyunggi 449-728, South Korea

E-mail: ehlmoon@mju.ac.kr 